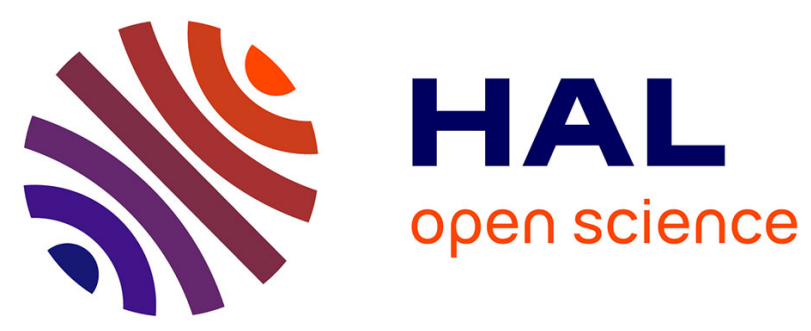

\title{
Sensitivity of Piecewise control laws with respect to perturbation of the state-space partition
}

\author{
Rajesh Koduri, Sorin Olaru, Pedro Rodriguez-Ayerbe
}

\section{To cite this version:}

Rajesh Koduri, Sorin Olaru, Pedro Rodriguez-Ayerbe. Sensitivity of Piecewise control laws with respect to perturbation of the state-space partition. The 21st International Conference on Control Systems and Computer Science (CSCS21), May 2017, Bucarest, Romania. 10.1109/CSCS.2017.9 . hal-01648113

\section{HAL Id: hal-01648113 \\ https://hal-centralesupelec.archives-ouvertes.fr/hal-01648113}

Submitted on 24 Nov 2017

HAL is a multi-disciplinary open access archive for the deposit and dissemination of scientific research documents, whether they are published or not. The documents may come from teaching and research institutions in France or abroad, or from public or private research centers.
L'archive ouverte pluridisciplinaire HAL, est destinée au dépôt et à la diffusion de documents scientifiques de niveau recherche, publiés ou non, émanant des établissements d'enseignement et de recherche français ou étrangers, des laboratoires publics ou privés. 


\title{
Sensitivity of Piecewise control laws with respect to perturbation of the state-space partition
}

\author{
Rajesh Koduri ${ }^{1}$ Sorin Olaru ${ }^{1}$ and Pedro Rodriguez-Ayerbe ${ }^{1}$
}

\begin{abstract}
The control design techniques for linear or hybrid systems (e.g. anti-wind up, MPC) lead often to off-line state-space partitions with non-overlapping convex polyhedral regions. This corresponds to a piecewise state feedback control laws associated to polyhedral partition of the state-space. In this work, we consider the perturbation in the representation of the vertices of the polyhedral regions. For such control laws, this problem is of particular interest with respect to the reduced precision representation of the state-space partition. The perturbed state-space partitions might lose one of the important property of the explicit controllers: the "non-overlapping" characterization. We derive a set called vertex sensitivity region to determine the admissible perturbation independently for each vertex of the polyhedral partition. A perturbation is deemed admissible if the non-overlapping property of the polyhedral regions is preserved. In the present work, the analysis of the sensitivity of each vertex is done under the assumption that the rest of the partition remains on the nominal configuration.
\end{abstract}

\section{INTRODUCTION}

Model Predictive Control (MPC) is one example of control design methodologies which has the ability to handle multivariate systems in the presence of constraints on inputs, states and outputs. MPC algorithms have been widely used to control plants or processes related to chemical, electrical and mechanical applications [1]. However, implementing an MPC algorithm to obtain the optimal control input on-line by solving a Quadratic Programming (QP) problem for every time step using the available measurement is time consuming, particularly when it comes to system with fast dynamics. This on-line computational complexity can be overcome by transforming the QP problem to multi-parametric Quadratic Programming (mp-QP) problem and solving it off-line at the control design stage. This approach of solving the mp-QP problem off-line is also called Explicit MPC (EMPC), where the computation of the optimal control input is reduced to a simple evaluation of algebraic functions stored in a look-up table [2], [3]. EMPC control laws can be easily evaluated and implemented on-line for systems with extremely fast dynamics and as long as the state-space models are of small dimensions.

Such control laws can be effectively implemented on micro-controller circuits or on Field-Programmable Gate

\footnotetext{
*This work is supported by TEMPO Marie-Curie European Project

${ }^{1}$ The Authors are with L2S, CentraleSupelec, CNRS, LIPS, Paris Saclay University, 91192, Gif-Sur-Yvette, France. rajesh.koduri@centralesupelec.fr, sorin.olarulcentralesupelec.fr, pedro.rodriguez@centralesupelec.fr
}

Array (FPGA) for a wide-range of control applications [4], [5]. The cost of the micro-controller circuits or FPGA depend on the size of the memory unit or Arithmetic and Logic Unit (ALU) associated with it. For storing a relatively small state-space partitions and the PWA control laws associated with each partition, the cost of the microcontroller required might be reasonable. But for storing considerably large state-space partitions and PWA functions, a truncation (interpreted also as a quantization) operation should be performed on the representation of the state-space regions and the associated PWA control laws in order to reduce the hardware costs [8]. The quantized state-space regions and quantized affine control laws associated with the regions adversely impact the accuracy of the control laws. Moreover, the modified regions might lose the nonoverlapping property of the state-space partition. Such a phenomenon will lead to non-uniqueness of the feedback control, discontinuities and the associated loss in stability, performance and other real-time certifications.

The authors of [6] proposed a geometrical approach to adopt the robustness margin for PWA control law with respect to the positive invariance characteristics of a nominal discrete-time linear system. This work has been extended in [7] to handle the robustness of the contractivity and not only the invariance. It is worth to be mentioned that these studies handle the robustness/fragility with respect to the model parameters and the control law gains but does not extend the analysis to the quantized explicit control law or quantized state-space regions. A recent study on the accuracy of explicit model predictive control law for quantized state-space partitions and quantized explicit control law is proposed in [8] analyzing in general the amount of bits required in order to achieve a relative degree of control input accuracy. However, the paper constructs the analysis based on the assumption that the quantized statespace regions are non-overlapping and thus do not answer to the essential question of the admissible representation errors in the state space domain. The present work deals with this essential property of non-overapping regions in the state space partitioning, thus completing all these previous studies on the rigorous theoretical ground.

In order to analyze the impact on the closed-loop, the present work starts from the existence of a discrete-time linear system controlled by an explicit piecewise affine control law. We show how the polyhedral partitions change when perturbations on the vertex representation of the poly- 
hedral partitions or regions take place. This perturbed vertex transforms the original state-space regions into new regions. The goal is to analyze to what extent the non-overlapping property of the polyhedral partition can be preserved in event of an perturbation in the representation of the vertices of the regions. We characterize the vertex perturbation by determining a set called vertex sensitivity region which preserves the non-overlapping property of the polyhedral partition.

The paper is organized as follows, After the definition of the basic notations, the background of the explicit MPC is presented and the main problem is stated in a mathematical form. In section III, the main results concerning with the formulation of overlapping in the change of partition is shown and the vertex sensitivity margin is provided with related theorem, proof and implementation algorithm. In section IV, the numerical example for vertex sensitivity is provided.

\section{BASic Notations AND PRELIMINARIES}

This section addresses some basic notations and definitions. The sets $\mathbb{R}, \mathbb{R}_{+}, \mathbb{Z}, \mathbb{N}$ and $\mathbb{N}_{+}$denote set of real numbers, set of non-negative real numbers, set of integers, set of non-negative integers, set of positive integers, respectively. We denote $\mathbb{R}^{n}$ a Euclidean space and $x \in \mathbb{R}^{n}$ a vector with $n$ elements.

A set $S \subset \mathbb{R}^{n}$ is a proper $\mathcal{C}$-set if it is convex, closed, compact and contains the origin in its interior. A polyhedron is the (convex) intersection of a finite number of open or closed half-spaces and a polytope is a bounded and closed polyhedron. The set of vertices of a polytope $M \subset \mathbb{R}^{n}$ is denoted $\mathcal{V}(M)$. For a $N \in \mathbb{N}_{+}, \mathcal{I}_{N}$ denotes the set of integers, $\mathcal{I}_{N}:=\left\{i \in \mathbb{N}_{+} \mid i \leq N\right\}$. For a given set $\mathcal{P}, \operatorname{int}(\mathcal{P})$ denotes the interior of $\mathcal{P}$ and Conv denotes the convex hull.

\section{BACKGROUND}

\section{A. System Description}

Let us consider for the statement of the problem a discretetime linear system given by,

$$
x(k+1)=A x(k)+B u(k),
$$

here, $x(k) \in \mathbb{R}^{n}$ is the state vector at time $k$ and $u(k) \in$ $\mathbb{R}^{m}$ is the control input vector. The system states and inputs variables are subject to constraints, with state constraints given by

$$
\mathcal{X}=\left\{x: H_{x} x \leq h_{x}, H_{x} \in \mathbb{R}^{p \times n}, h_{x} \in \mathbb{R}^{p}\right\},
$$

And input constraints by,

$$
\mathcal{U}=\left\{u: H_{u} u \leq h_{u}, H_{u} \in \mathbb{R}^{p_{u} \times m}, h_{u} \in \mathbb{R}^{p_{u}}\right\} .
$$

where the matrices $H_{x}, H_{u}$ and the vectors $h_{x}, h_{u}$ are assumed to be constant, and $\mathcal{X} \subset \mathbb{R}^{n}$ and $\mathcal{U} \subset \mathbb{R}^{m}$. The state and input constraints sets $\mathcal{X}$ and $\mathcal{U}$ are proper $\mathcal{C}$-sets.
Definition 1: A closed and bounded set $\mathcal{P} \subset \mathcal{X}$ is called controlled positively invariant with respect to (1) if there exists a control law $u^{*}(x(k))$, such that $\forall x(0) \in \mathcal{P}$, then $x(k) \in \mathcal{P}, \forall k \in \mathbb{Z}$.

Consider the discrete-time linear prediction model given by (1) subject to the state and input constraints (2), (3). The constrained finite-time optimal control problem for the standard MPC is given by,

$$
\begin{gathered}
J(x(k), U)=\min _{u \in U} x\left(k+N_{p}\right)^{T} P x\left(k+N_{p}\right)+ \\
\sum_{j=0}^{N_{p}-1}\left(x(k+j)^{T} Q x(k+j)+u(k+j)^{T} R u(k+j)\right) \\
\text { s.t. } \quad x(k+j+1)=A x(k+j)+B u(k+j) \\
j=0, \cdots, N_{p}-1 \\
x(0)=x(k), \quad x\left(N_{p}\right) \in \mathcal{X}_{f} \\
u(k+j) \in \mathcal{U}, \quad x(k+j) \in \mathcal{X}, j=0, \cdots, N_{p}-1 .
\end{gathered}
$$

Here, $N_{p}$ is the prediction horizon and the optimal control sequence obtained from the open-loop finite-time optimal control problem can be written as:

$$
U^{*}=\left[u(0)^{* T} \cdots u\left(N_{p}-1\right)^{* T}\right]^{T} \in \mathbb{R}^{m} .
$$

The weighting matrices $Q=Q^{T} \geq 0$ and $P=P^{T} \geq 0$ are positive semi-definite matrices, and $R=R^{T}>0$ is a positive definite matrix, all of them contributing to the definition of the performance index of the optimization problem. $\mathcal{X}_{f}$ is a controlled invariant set admissible with respect to the input and state constraints [10].

The MPC problem (4) can be equivalently written in terms of a multi-parametric Quadratic Programming (mpQP) problem [3].

$$
\begin{array}{r}
J\left(x, U^{*}\right)=\min _{U^{*}} \frac{1}{2} U^{* T} \bar{H} U^{*}+x^{T} \bar{F}^{T} U^{*}+\frac{1}{2} x^{T} \bar{Y} x, \\
\text { s.t } \quad \bar{G} U^{*} \leq \bar{W}+\bar{E} x .
\end{array}
$$

In (6), $x$ is the current state supposed to be measured. The matrices $\bar{H}, \bar{F}, \bar{Y}, \bar{G}, \bar{W}, \bar{E}$ are obtained after the transformation (see [10] for further details).

Definition 2: Consider a set of sets $\mathcal{P}_{N}(R)$. This will define a polyhedral partition of the $\mathcal{C}$-set $R \subset \mathbb{R}^{n}$, with $\mathcal{P}_{N}(R)=\left\{R_{1}, R_{2} \cdots R_{N}\right\}, N \in \mathbb{N}_{+}$and $R_{i} \subset \mathbb{R}^{n}$ if

1) $R=\cup_{i \in \mathcal{I}_{N}} R_{i}$,

2) $\operatorname{int}\left(R_{i}\right) \neq \emptyset, \forall i \in \mathcal{I}_{N}$,

3) $\operatorname{int}\left(R_{i}\right) \cap \operatorname{int}\left(R_{j}\right)=\emptyset, \forall(i, j) \in \mathcal{I}_{N}^{2}$, and $i \neq j$.

Definition 3: The solution to the EMPC problem is a piecewise affine function $f_{\text {pwa }}$ defined over a polyhedral partition $\mathcal{P}_{N}(R)$ :

$$
f_{p w a}(x)=F_{i} x_{k}+g_{i}, \text { if } x_{k} \in R_{i} .
$$

with $i \in \mathcal{I}_{N}, F_{i} \in \mathbb{R}^{m \times n}$ and $g_{i} \in \mathbb{R}^{m}$, and the regions $R_{i}$ are polytopic and bounded.

The feedback law (7) is continuous and by substituting in the equation (1), it leads to the PWA closed-loop dynamics:

$$
x(k+1)=\left(A+B F_{i}\right) x(k)+B g_{i} \forall x(k) \in R_{i}, \quad i \in \mathcal{I}_{N} .
$$




\section{MAIN RESULtS}

The polyhedral partition $\mathcal{P}_{N}(R)$ considered next is the one resulting from the solution of the EMPC problem (6). In order to develop the sensitivity analysis, let us consider a polytopic region $R_{i} \subset R \in \mathbb{R}^{n}$ within the polyhedral partition given by the half space representation,

$$
R_{i}=\left\{x \mid H_{i} x \leq k_{i}\right\}, \quad i \in \mathcal{I}_{\mathcal{N}}
$$

For each such region, an equivalent vertex representation exists in the virtue of Motzkin duality [11]:

$$
R_{i}=\operatorname{Conv}\left\{v_{i, 1}, \cdots, v_{i, r_{i}}\right\}, i \in \mathcal{I}_{N},
$$

where $r_{i}$ is the number of vertices of $R_{i}$.

In order to analyze the sensitivity of this set representation and its implication on the PWA control, a perturbation in the representation of the vertex $v_{i, j}, j \in \mathcal{I}_{r_{i}}$ of the region $\hat{R}_{i}$ :

$$
\hat{v}_{i, j}=v_{i, j}+\Delta v_{i, j}
$$

will be considered leading to a new polyhedral set:

$$
\hat{R}_{i}=\operatorname{Conv}\left\{v_{i, 1}, v_{i, 2}, \ldots, v_{i, j}+\Delta v_{i, j}, \cdots, v_{i, r_{i}}\right\} .
$$

The comparative analysis of the set (9) and (10) with respect to (12) will represent the main object of study.

Remark: The analysis will privilege the vertex representation (11) with respect to the half-space representation (9) for two reasons. The first is related to the fact that the perturbation is concentrated on a single element of the set of generators with obvious computation advantages. A second argument is related to the non-emptiness of the set $\hat{R}_{i}$ in (12) independent on the magnitude of the perturbation $\Delta v_{i, j}$. As a term of comparison, the equivalent half-space representation of $\hat{R}_{i}$ is

$$
\hat{R}_{i}=\left\{x \mid \hat{H}_{i} x \leq \hat{k}\right\}, i \in \mathcal{I}_{\mathcal{N}},
$$

where several entries of the inequalities are affected and thus the analysis of the perturbation will have to deal with structural problems with respect to the Definition 2 of the polyhedral partitions. Indeed considering a perturbation directly with respect to the half-space representation (9) can lead to an empty set $\hat{R}_{i}$ posing well-possessedness issues in the characterization of the partition.

With respect to the perturbed sets in (12), the main issue is the overlapping of the regions occurred due to representation errors in the original vertices of state-space partitions.

In order to provide a graphical interpretation of the problem under study let us consider the example of a simple continuous PWA function $f_{\text {pwa }}(x)$ defined over the interval $[-\mu, \mu]=\left[v_{2,1}, v_{3,2}\right] \subset R$.

$$
f_{\text {pwa }}(x)=\left\{\begin{array}{lll}
\alpha x & \text { for } & x \in R_{1}=\operatorname{Conv}\left\{v_{1,1}, v_{1,2}\right\} \\
\bar{\beta} & \text { for } & x \in R_{2}=\operatorname{Conv}\left\{v_{2,1}, v_{2,2}\right\} \\
\bar{\gamma} & \text { for } & x \in R_{3}=\operatorname{Conv}\left\{v_{3,1}, v_{3,2}\right\}
\end{array}\right\}
$$

which can be simplified by the continuity between the neighboring regions: $v_{1,2}=v_{3,1}$ and $v_{2,2}=v_{1,1}$.

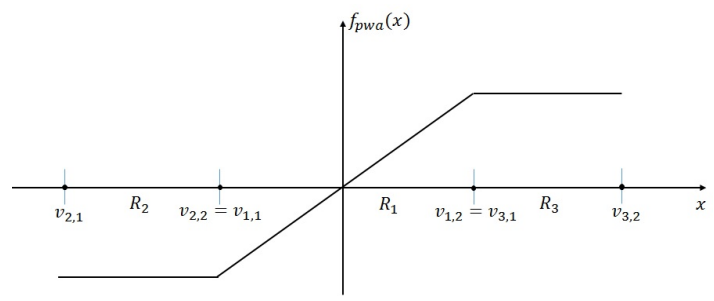

(a) 1-D Polyhedral with three regions $R_{1}, R_{2}$ and $R_{3}$ and the corresponding PWA functions before perturbation.

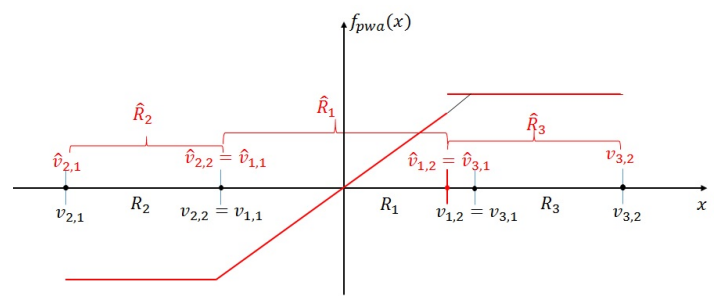

(b) 1-D Polyhedral with three regions $R_{1}, R_{2}$ and $R_{3}$ before perturbation of the vertex representation. Illustration of regions $\hat{R}_{1}, \hat{R}_{2}$ and $\hat{R}_{3}$ after perturbation preserving the property, $\operatorname{int}\left(\hat{R}_{i}\right) \cap \operatorname{int}\left(\hat{R}_{j}\right)=\emptyset, \forall i, j \in$ $\mathcal{I}_{3}, i \neq j$.

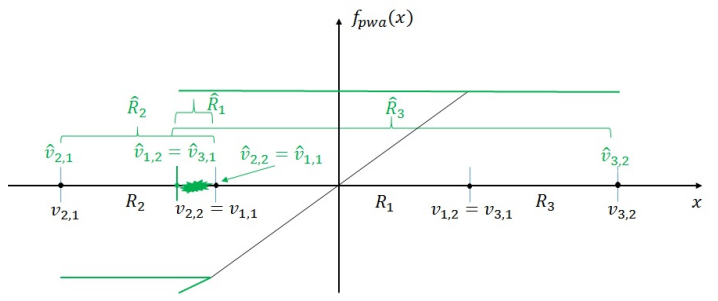

(c) 1-D Polyhedral with three regions $R_{1}, R_{2}$ and $R_{3}$ before perturbation. Illustration of the regions $\hat{R}_{1}, \hat{R}_{2}$ and $\hat{R}_{3}$ after perturbation with the particular configuration leading to: $\operatorname{int}\left(\hat{R}_{i}\right) \cap i n t\left(\hat{R}_{j}\right) \neq \emptyset, \forall i, j \in \mathcal{I}_{3}, i \neq$ $j$.

Fig. 1: 1-D Polyhedral partition with three regions before and after perturbation and the corresponding PWA function.

In Fig. 1, the regions representation before and after the perturbation on the vertices is presented. In Fig. 1 (a), the nominal three regions obtained from EMPC denoted by $R_{1}, R_{2}$ and $R_{3}, R=R_{1} \cup R_{2} \cup R_{3}$ satisfy by definition the property $\operatorname{int}\left(R_{i}\right) \cap \operatorname{int}\left(R_{j}\right)=\emptyset, \forall i, j \in \mathcal{I}_{3}, i \neq j$.

The vertices of the regions are denoted by $v_{i, r_{i}}$, here $i$ and $r_{i}$ denote the index of regions and the index of vertices for each region respectively. After introducing a perturbation $\Delta v$ on the vertex $v_{1,2}$ and $v_{3,1}$ (recall that $\left.v_{1,2}=v_{3,1}\right)$ which falls in the regions $R_{1}$ and $R_{3}$, the perturbed vertex is denoted by $\hat{v}_{1,2}$ and $\hat{v}_{3,1}\left(\hat{v}_{1,2}=v_{1,2}+\Delta v_{1,2}, \hat{v}_{3,1}=v_{3,1}+\Delta v_{3,1}\right.$, $\left.\hat{v}_{1,2}=\hat{v}_{3,1}\right)$ as depicted in Fig. 1 (b).

Consequently, one vertex displacement will influence the topology of the regions $R_{1}$ and $R_{3}$ and leading to $\hat{R}_{1}$ and $\hat{R}_{3}$, while region $R_{2}=\hat{R}_{2}$ remains unchanged. It can be noticed from Fig. 1 (b) that even after the change in the regions, $\hat{R}=\hat{R}_{1} \cup \hat{R}_{2} \cup \hat{R}_{3}$ and particularly the property $\operatorname{int}\left(\hat{R}_{i}\right) \cap$ $\operatorname{int}\left(\hat{R}_{j}\right)=\emptyset, \forall i, j \in \mathcal{I}_{3}, i \neq j$ is preserved. Thus the nonoverlapping property of the polyhedral partition holds.

A very important consequence of the perturbation is the 
lost of the continuity for the PWA function $f_{\text {pwa }}(x)$ defined over $R=\hat{R}_{1} \cup \hat{R}_{2} \cup \hat{R}_{3}$. Moreover, the control input depends on which region the state vector $x$ falls into and in particular with the regions $\hat{R}_{1}$ and $\hat{R}_{3}$, the control action being multivalued at the frontier. It is obvious that the control input modifications will lead to a loss of control continuity and consequently of the performances. This phenomenon can be seen however as a singularity and is out of the scope of the present work.

In the same framework, in Fig. 1 (c), the vertex $v_{1,2}$ and $v_{3,1}$ is perturbed to $\hat{v}_{1,2}$ and $\hat{v}_{3,1}$ and jumped into the region $R_{2}$ adversely affecting all the three regions. The new regions denoted by $\hat{R}_{1}, \hat{R}_{2}$ and $\hat{R}_{3}$ are overlapping, i.e., $\operatorname{int}\left(\hat{R}_{1}\right) \cap \operatorname{int}\left(\hat{R}_{2}\right) \neq \emptyset$, $\operatorname{int}\left(\hat{R}_{2}\right) \cap \operatorname{int}\left(\hat{R}_{3}\right) \neq \emptyset$ and $\operatorname{int}\left(\hat{R}_{1}\right) \cap \operatorname{int}\left(\hat{R}_{3}\right) \neq \emptyset$.

This possible overlapping due to changes in the vertices of the polyhedral partition represents a critical structural change because the unicity of the control law is lost on a compact full-dimensional region of the state space. The nonuniqueness of the control action leads to behaviors which are difficult to characterized in terms of determinedness and lose of performance and thus should be avoided in the first place. This issue forms the basis for investigation in the present work and can be resumed by the need to characterize the limits of the perturbation which preserve the "non-overlapping" property of the polyhedral partition.

In the following, a formal definition of the vertex sensitivity is provided in the perspective of non-overlapping property of the polyhedral regions.

Definition 4: Consider the polyhedral partition $\mathcal{P}_{N}(R) \in$ $\mathbb{R}^{n}$ with $R_{i}=\operatorname{Conv}\left\{v_{i, 1}, \cdots, v_{i, r_{i}}\right\}, i \in \mathcal{I}_{N}$. Let $v \in \mathbb{R}^{n}$ be a vertex within the polyhedral partition $\mathcal{P}_{N}(R)$ and denote $J^{v}$ as the subset of indexes of regions having $v$ as a vertex:

$$
J^{v}=\left\{j \in \mathcal{I}_{N} \mid v \in \mathcal{V}\left(R_{j}\right)\right\}
$$

The set $\hat{V} \subset \mathbb{R}^{n}$ is describing the vertex sensitivity region for $v$ if $\forall \hat{v} \in \hat{V}$ the collection of sets

$$
\left\{\begin{array}{l}
\hat{R}_{j}=\operatorname{Conv}\left\{\mathcal{V}\left(R_{j}\right) \backslash\{v\}, \hat{v}\right\}, \forall j \in J^{v}, \\
\hat{R}_{j}=R_{j}, \forall j \in \mathcal{I}_{N} \backslash J^{v}
\end{array}\right.
$$

represents a polyhedral partition: $\hat{\mathcal{P}}_{N}(R)=\left\{\hat{R}_{1}, \cdots, \hat{R}_{N}\right\}$.

Theorem 1: Consider the subset of regions $R_{j}, j \in J^{v}$ of $\mathcal{P}_{N}(R)$ such that $v \in \mathcal{V}\left(R_{j}\right), \forall j \in J^{v}$, then the perturbed vertex sensitivity is represented by a polyhedral set $\hat{V} \subset \mathbb{R}^{n}$.

Proof: The proof will be constructive based on the enumeration of the possible overlapping scenarios and superposing the linear constraints imposed to avoid such configurations. The indexes within $J^{v}$ identify the regions containing $v$ as a vertex. For each $j \in J^{v}$, the half-space representation can be exploited:

$$
\begin{array}{r}
R_{j}=\left\{x \mid H_{j} x \leq k_{j}\right\} \quad \text { or explicitly } \\
R_{j}=\left\{x \mid h_{j, r_{i}} x \leq k_{j, r_{i}}, r_{i}=1, \cdots, r_{j}\right\}
\end{array}
$$

here $r_{j}$ is the total number of closed half-spaces of $R_{j}$. It is straight forward to identify the subset of those half-spaces passing through the vertex $v$.

Consider $n_{v}$ to be the total number of hyperplanes $h_{j, r_{i}} x=k_{j, r_{i}}, j \in J^{v}, r_{i}=\left\{1, \cdots, r_{j}\right\}$ from the closed half-spaces that construct the regions $R_{j}, \forall j \in J^{v}$ based on the definition (17b). Let us denote $m_{v}$ with $m_{v}<n_{v}$ the number of hyperplanes that pass through the vertex $v$. Let us reorder the complete set of half-spaces of $R_{j}, \forall j \in J^{v}$ such that the first $m_{v}$ pass by $v$. We have then $h_{i} v=k_{i}, \forall i \in \mathcal{I}_{m_{v}}$.

Scenario I: Let us construct a set denoted as $\tilde{R}$ which includes a first set of constraints imposed on the vertex,

$$
\tilde{R}=\left\{x \in \mathbb{R}^{n} \mid h_{i} x \leq k_{i}, \forall i \in \mathcal{I}_{m_{v}} \backslash \mathcal{I}_{n_{v}}\right\} .
$$

The set $\tilde{R} \subset \bigcup_{j \in J^{v}} R_{j}$ is a closed convex subset of the union of the regions $R_{j}$. Supposing that $\hat{v} \notin \tilde{R}$, then the perturbed vertex characterize an overlapping phenomena. Such excessive perturbation leads to $\hat{v} \notin \bigcup_{j \in J^{v}} R_{j}$ and $\hat{v} \in \bigcup_{j \in \mathcal{I}_{N} \backslash J^{v}} R_{j}$ and this overlapping violation makes some closed half-spaces of the regions $R_{j}$ redundant. Considering a case $\hat{v} \notin \bigcup_{j \in J^{v}} R_{j}$ and $\hat{v} \notin \bigcup_{j \in \mathcal{I}_{N} \backslash J^{v}} R_{j}$, the perturbed vertex implodes the feasible set $R$ and such violations will lead to the invalidation of the polyhedral partition $\mathcal{P}_{N}(R)$. There after, in (18) some constraints are placed to make the set $\tilde{R}$ closed and bounded and positioning the perturbed vertex such that $\hat{v} \in \tilde{R}$ will overcome such possibilities of overlapping phenomena.

Scenario II: Let us introduce the set $\Phi$ and initialize it $\Phi \leftarrow \mathbb{R}^{n}$. Consider a subset of the regions $R_{j}$ and denote it by $\bar{R}_{j}, \forall j \in J^{v}$,

$$
\bar{R}_{j}=\operatorname{Conv}\left\{\left\{v_{j, 1}, \cdots, v_{j, r_{j}}\right\} \backslash v\right\} .
$$

By duality, the set $\bar{R}_{j}$ is equivalently given by the half-space representation:

$$
\bar{R}_{j}=\left\{x \mid \bar{h}_{j, r_{b}} x \leq \bar{k}_{j, r_{b}}, r_{b}=1, \cdots, \bar{r}_{j}\right\}
$$

here, $\bar{r}_{j}$ denotes the total number of closed half-space inequalities of the region $\bar{R}_{j}$. In the following, we aim to impose a set of linear constraints on $\hat{v}$ to avoid the overlapping violations caused by the modifications on the $m_{v}$ number of hyperplanes that pass through the vertex $v$ from (18). Consider $\bar{n}_{v}$ be the total number of closed halfspaces from the regions $\bar{R}_{j}, \forall j \in J^{v}$ as defined in (20). Now analyzing each of the $\bar{n}_{v}$ closed half-spaces with respect to the closed and bounded set $\tilde{R}$ one can construct the set:

$\Phi=\left\{\tilde{R} \cap\left\{-\bar{h}_{i} x \leq-\bar{k}_{i}\right\}, \forall i \in \mathcal{I}_{\bar{n}_{v}} \tilde{R} \cap\left\{\bar{h}_{i} x \leq \bar{k}_{i}\right\} \neq \tilde{R}\right\}$.

Whenever a perturbation takes place such that $\hat{v} \in \operatorname{int}\left(\bar{R}_{j}\right)$ and $\hat{v} \in \tilde{R}$, for some $j \in J^{v}$, it leads to an overlapping scenario. Indeed the regions $\hat{R}_{j}=\bar{R}_{j}$ for all the indexes $j$ such that $\hat{v} \in \operatorname{int}\left(\bar{R}_{j}\right)$. All the remaining regions with indexes $j$ such that $\hat{v} \notin \operatorname{int}\left(\bar{R}_{j}\right)$ are containing the point $\hat{v}$ as a vertex and the overlapping is proved. 

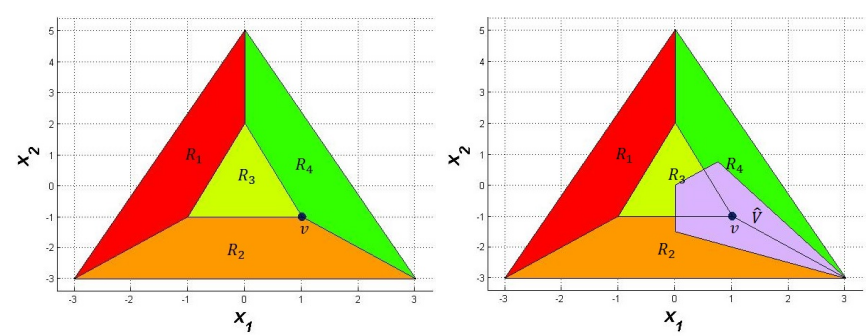

(a) Polyhedral with four regions (b) The vertex of interest $v$ and $R_{1}, R_{2}, R_{3}, R_{4}$ and the black dot the vertex sensitivity region $\hat{V}$ is represents $v$. shown.

Fig. 2: Polyhedral partition with four regions, the vertex of interest $v$ and the vertex sensitivity region $\hat{V}$ are shown.
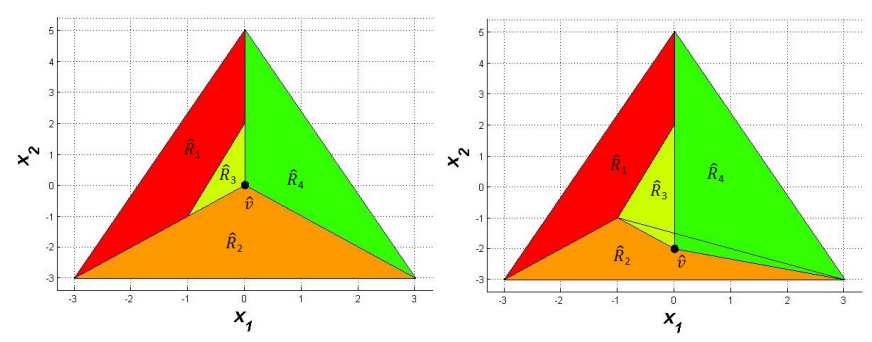

(a) After perturbation $\hat{v} \in \hat{V}$ (b) After perturbation $\hat{v} \notin \hat{V}$ the regions are changed and $\operatorname{int}\left(\hat{R}_{i}\right) \cap \operatorname{int}\left(\hat{R}_{j}\right) \neq \emptyset, \forall i, j \in$ $\operatorname{int}\left(\hat{R}_{i}\right) \cap \operatorname{int}\left(\hat{R}_{j}\right)=\emptyset, \forall i, j \in J^{v}, i \neq j$.

$J^{v}, i \neq j$.

Fig. 3: The vertex $v$ denoted by a black dot in Fig. 2 (a) is perturbed to $\hat{v}$ changing the regions $R_{1}, R_{2}, R_{3}, R_{4}$ to $\hat{R}_{1}, \hat{R}_{2}, \hat{R}_{3}, \hat{R}_{4}$.

To avoid this phenomenon, the restriction to the region $\Phi$ described in (21) needs to be imposed in order to avoid $\hat{v} \in \operatorname{int}\left(\bar{R}_{j}\right)$.

Finally, the set that describes for all possible admissible perturbation for a given vertex $v$ preserving the overlapping property of the polyhedral partition can be given as:

$$
\hat{V} \leftarrow \Phi \text {. }
$$

Fig. 2 (a) shows a polyhedral partition with four regions $R_{i}, i=1, \cdots, 4$ and the vertex of interest $v=\left[\begin{array}{ll}1 & -1\end{array}\right]^{T}$ denoted by a black dot. The vertex $v$ is one of the generators of three among the four regions of the partition. In Fig. 2 (b), the sensitivity of the vertex $v$ is represented by $\hat{V}$ with a blue polytope. By consequence the vertex denoted by $\hat{v}$ must be settled to any of the points in the polytope $\hat{V}$ in the event of loss of precision in the representation.

The vertex sensitivity region $\hat{V}$ is constrained by four closed half-space inequalities. For any point outside the blue polytope, the non-overlapping property of the EMPC is lost. The new regions formed with the displaced vertex $\hat{v} \in \hat{V}$ guarantees the "non-overlapping" property of the polyhedral partition. This observation is better validated with the help of the Fig. 3 (a) and Fig. 3 (b), where the polyhedral regions are recreated by the displacement of vertex $\hat{v}$. In Fig. 3 (a) the vertex $v=[1-1]^{T}$ is displaced to vertex $\hat{v}=\left[\begin{array}{ll}0 & 0\end{array}\right]^{T} \in \hat{V}$ which alters all the four regions and still preserves the overlapping property i.e., $\operatorname{int}\left(\hat{R}_{i}\right) \cap \operatorname{int}\left(\hat{R}_{j}\right)=$ $\emptyset, \forall i, j \in \mathcal{I}_{4}, i \neq j$. In Fig. 3 (b), it is clearly visible that the overlapping of the regions takes place since $\hat{v}=\left[\begin{array}{ll}0 & -2\end{array}\right]^{T} \notin$ $\hat{V}$.

The procedure for computing the vertex sensitivity region $\hat{V}$ is resumed in Algorithm 1.

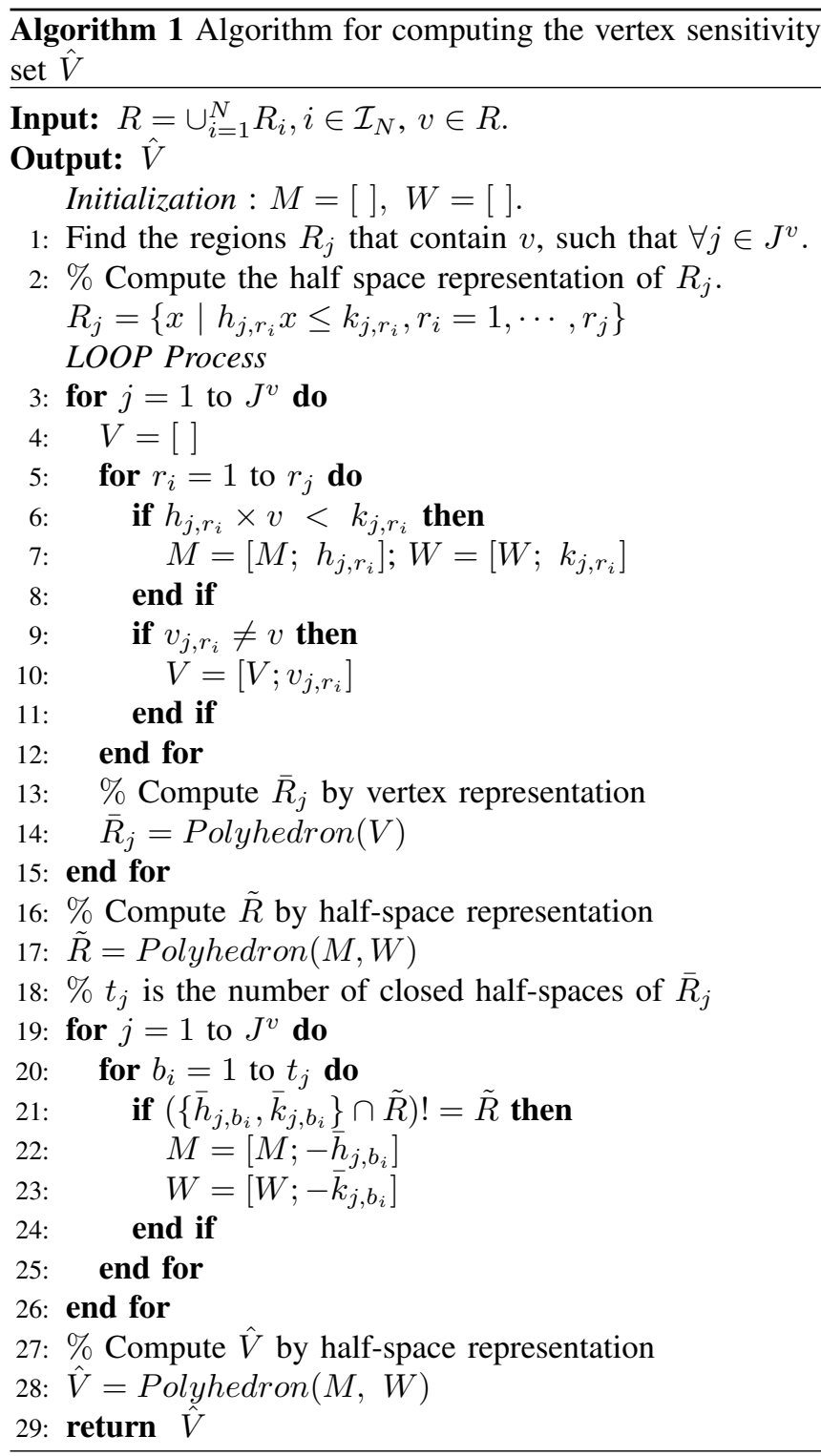

\section{NumERICAL EXAMPLES}

Consider a discrete-time linear system,

$x(k+1)=\left[\begin{array}{cc}1.4 & 0 \\ 0.8 & -1.1\end{array}\right] x(k)+\left[\begin{array}{l}0.5 \\ 0.7\end{array}\right] u(k)$.

Consider the finite time optimal control based on quadratic cost with weighting matrices $Q=\left[\begin{array}{ll}1 & 0 \\ 0 & 1\end{array}\right]$ and $R=1$. 

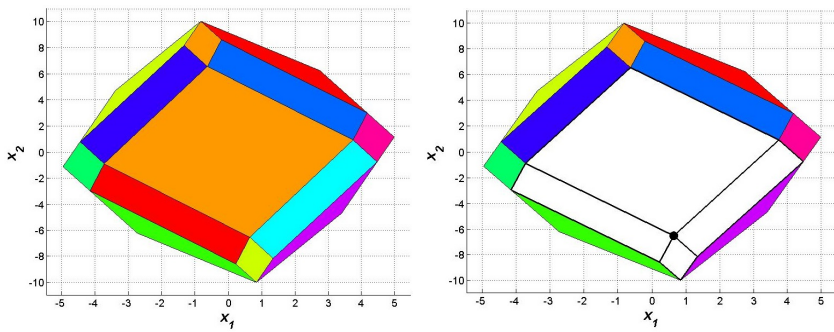

(a) State-space partition with 13 (b) The vertex of interest $v$ regions.

shown with a black dot and the polyhedrals containing $v$ is colored with white.

Fig. 4: State-space partition with 13 regions

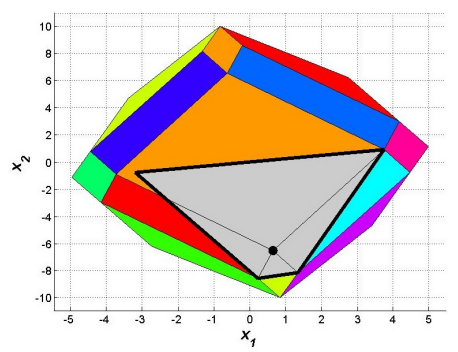

Fig. 5: Vertex sensitivity region $\hat{V}$ is represented by a gray polytope.

The prediction horizon chosen is 2 , the input constraint $-5 \leq u(k) \leq 5$ and the state constraint $-5 \leq[10] x(k) \leq 5$ are considered in the receding horizon optimization. Solving the EMPC problem yields 13 affine controllers and their validity regions obtained using MPT Toolbox 3.0 to compute the polyhedral regions and their associated PWA control laws [12].

Fig. 4 (a), presents the state-space partition $\mathcal{P}_{N}(R)$ with 13 regions denoted as $R=\cup_{i=1}^{13} R_{i}$ each of it associated with a gain matrix $F_{i}$ and an offset $g_{i}$. In Fig. 4 (b), the vertex of interest $v$ is denoted by a black dot and the regions $R_{j}, j \in J^{v}$ containing the point $v$ are highlighted in terms of a union of polyhedra depicted in white color. By applying the main result of the present paper and the Algorithm 1, the vertex sensitivity region is obtained as presented in Fig. 5 in gray color. In Fig. 6 (a), the vertex $v$ is displaced to $\hat{v}$ within the sensitivity region $\hat{V}$ affecting the shapes of four regions and the perturbed collection of regions $\hat{R}_{j}, j=1, \cdots, 13$ does not violate the non-overlapping property. The plot is recreated with four changed regions whereas the unaffected regions kept unchanged.

In Fig. 6 (b), we can see the overlapping takes places among the affected regions caused by a perturbation in the vertex which exceeded the admissible sensitivity margin for the respective vertex $\hat{v} \notin \hat{V}$.

\section{CONCLUSIONS}

In the present work, we derived a vertex sensitivity set characterizing the admissible disturbance with respect to a given vertex all by preserving the non-overlapping property
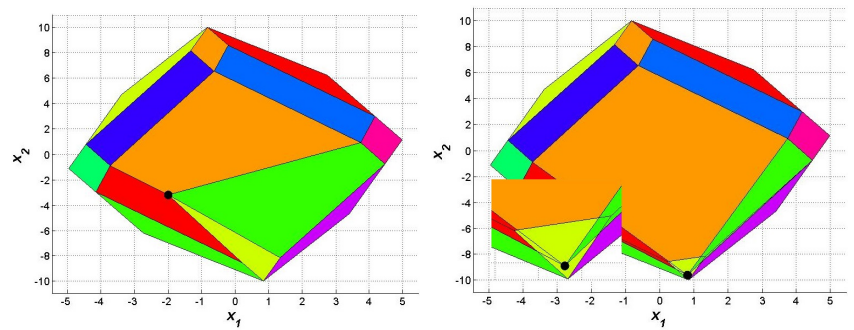

(a) The vertex $\hat{v} \in \hat{V}$ preserves (b) The vertex $\hat{v} \notin \hat{V}$ violates the non-overlapping property of the non-overlapping property of the regions.

the regions, the zoomed part confirms the violation does happen.

Fig. 6: The black dot in both figures show the perturbed vertex $\hat{v}$.

of the polyhedral regions. This element is fundamental in the characterization of the sensitivity of the representation of a polyhedral partition. The next step towards this goal is to consider simultaneous disturbance in all the vertices of the polyhedral partition. Further work is needed to analyze the condition such that the trajectories converge to the origin despite the perturbation on the vertex of the polyhedral regions.

\section{REFERENCES}

[1] D. Q. Mayne, J. B. Rawlings, C. V. Rao, and P. O. M. Scokaert, Constrained model predictive control: Stability and optimality, Automatica, vol. 36(6), pp. 789-814, 2000.

[2] A. Alessio and A. Bemporad, A survey on explicit model predictive control, In Nonlinear model predictive control: Springer 2009, pp. 345-369.

[3] A. Bemporard, M. Morrari, V. Dua and E. N. Pistikopoulos, The explicit linear quadratic regulator for constrained systems, Automatica, vol. 38(1), pp. 3-20, 2002.

[4] T. A. Johansen, W. Jackson, R. Schreiber and P. Tondel, Hardware Synthesis of Explicit Model Predictive Controllers, IEEE Transactions on control systems technology, vol. 15, ,no. 1, Jan 2007.

[5] D. Ingole, M. Kvasnica, FPGA Implementation of Explicit Model Predictive Control for Closed Loop Control of Depth of Anesthesia, Preprints, 5th IFAC Conference on Nonlinear Model Predictive Control, Spain, September. 2015.

[6] N. A. Nguyen, S. Olaru, P. Rodrguez-Ayerbe, G. Bitsoris and M. Hovd, Explicit robustness and fragility margins for linear discrete systems with piecewise affine control law, Automatica, vol. 68, pp. 334-343, June, 2016.

[7] R. Koduri, P. Rodriguez-Ayerbe and S. Olaru, Robustness margin for piecewise affine explicit control law, 55th IEEE Conference on Decision and Control, USA, December, 2016.

[8] A. Knyazev, P. Zhu, S. Di Cairano, Explicit model predictive control accuracy analysis, 54th IEEE Conference on Decision and Control (CDC), Osaka, pp. 2389-2394, December. 2015.

[9] E. G. Gilbert and K. T. Tan, Linear systems with state and control constraints: The Theory and Application of Maximal Output Admissible Sets, IEEE transactions on Automatic Control, vol. 36(9), September, 1991.

[10] S. Olaru and D. Dumur, Avoiding constraints redundancy in predictive control optimization routines, IEEE Transactions on Automatic Control, vol. 50(9), pp. 1459-1465, 2005.

[11] T. S. Motzkin, H. Raiffa, G. L. Thompson, and R. M. Thrall, The double description method, Princeton: Princeton Uni. Press, 1953.

[12] M. Herceg and M. Kvasnica and C. N. Jones and M. Morari, MultiParametric Toolbox 3.0, Proc. of the European Control Conference, pp. 502-510, July 2013. 